\title{
STRONG TIME OPERATORS ASSOCIATED WITH GENERALIZED HAMILTONIANS
}

\author{
Fumio Hiroshima*† Sotaro Kuribayashi ${ }^{\ddagger}$ \\ and Yasumichi Matsuzawa ${ }^{\S}$
}

\begin{abstract}
Let the pair of operators, $(H, T)$, satisfy the weak Weyl relation:

$$
T e^{-i t H}=e^{-i t H}(T+t),
$$

where $H$ is self-adjoint and $T$ is closed symmetric. Suppose that $g$ is a realvalued Lebesgue measurable function on $\mathbb{R}$ such that $g \in C^{2}(\mathbb{R} \backslash K)$ for some closed subset $K \subset \mathbb{R}$ with Lebesgue measure zero. Then we can construct a closed symmetric operator $D$ such that $(g(H), D)$ also obeys the weak Weyl
\end{abstract} relation.

\section{Weak Weyl relation and strong time operators}

\section{$1.1 \quad$ Introduction}

The energy of a quantum system can be realized as a self-adjoint operator on some Hilbert space, whereas time $t$ is treated as a parameter, and not intuitively as an operator. So, since the foundation of quantum mechanics, the energy-time uncertainty relation has had a different basis from that underlying the position-momentum uncertainty relation.

Let $Q$ be the multiplication operator defined by $(Q f)(x)=x f(x)$ with maximal domain $\mathrm{D}(Q)=\left\{\left.f \in L^{2}(\mathbb{R})\left|\int\right| x\right|^{2}|f(x)|^{2} d x<\infty\right\}$ and let $P=-i d / d x$ be the weak derivative with domain $H^{1}(\mathbb{R})$. In quantum mechanics, the position operator $Q$ and

\footnotetext{
${ }^{*}$ F.H. thanks for Grant-in-Aid for Science Research (B) 20340032 from JSPS for financial support.

${ }^{\dagger}$ Graduate School of Mathematics, Kyushu University 812-8581, Fukuoka, Japan

${ }^{\ddagger}$ Graduate School of Mathematics, Kyushu University 812-8581, Fukuoka, Japan

$\S$ Department of Mathematics, Hokkaido University, Sapporo, 060-0810, Japan
} 
the momentum operator $P$ in $L^{2}(\mathbb{R})$ obey the Weyl relation: $e^{-i s P} e^{-i t Q}=e^{i s t} e^{-i t Q} e^{-i s P}$ for $s, t \in \mathbb{R}$. From this we can derive the so-called weak Weyl relation:

$$
Q e^{-i t P}=e^{-i t P}(Q+t), \quad t \in \mathbb{R}
$$

and moreover the canonical commutation relation $[P, Q]=-i I$ also holds. The strong time operator $T$ is defined as an operator satisfying (1.1) with $Q$ and $P$ replaced by $T$ and the Hamiltonian $H$ of the quantum system under consideration, respectively.

More precisely, we explain the weak Weyl relation (1.1) as follows. Let $\mathscr{H}$ be a Hilbert space over the complex field $\mathbb{C}$. We denote by $\mathrm{D}(L)$ the domain of an operator $L$.

Definition 1.1 We say that the pair $(H, T)$ consisting of a self-adjoint operator $H$ and a symmetric operator $T$ on $\mathscr{H}$ obeys the weak Weyl relation if and only if, for all $t \in \mathbb{R}$

(1) $e^{-i t H} \mathrm{D}(T) \subset \mathrm{D}(T)$;

(2) $T e^{-i t H} \Phi=e^{-i t H}(T+t) \Phi$ for all $\Phi \in \mathrm{D}(T)$.

Here $T$ is referred to as a strong time operator associated with $H$ and we denote it by $T_{H}$ for $T$. Note that a strong time operator is not unique. Although from the weak Weyl relation it follows that $\left[H, T_{H}\right]=-i I$, the converse is not true; a pair $(A, B)$ satisfying $[A, B]=-i I$ does not necessarily obey the Weyl relation or the weak Weyl relation. If strong time operator $T_{H}$ is self-adjoint, then it is known that

$$
e^{-i s T_{H}} e^{-i t H}=e^{-i s t} e^{-i t H} e^{-i s T_{H}}
$$

holds. In particular when Hilbert space $\mathscr{H}$ is separable, by the von Neumann uniqueness theorem the Weyl relation (1.2) implies that $H$ and $T_{H}$ are unitarily equivalent to $\oplus^{n} P$ and $\oplus^{n} Q$ with some $n$, respectively. This asserts that any strong time operators associated with a semibounded $H$ on a separable Hilbert space are symmetric non-self-adjoint. These facts may implicitly suggest that strong time operators are not "observable".

A time operator but not necessarily strong associated with a self-adjoint operator $H$ is defined as an operator $T$ for which $[H, T]=-i I$. As was mentioned above, although a strong time operator is automatically a time operator, the converse is not true. It is remarkable that when the pair $(H, T)$ obeys the weak Weyl relation, $H$ has 
purely absolutely continuous spectrum. For example there is no strong time operator associated with the harmonic oscillator $\frac{1}{2}\left(P^{2}+\omega^{2} Q^{2}\right)$, whereas its time operator is formally given by

$$
\frac{1}{2 \omega}\left(\arctan \left(\omega P^{-1} Q\right)+\arctan \left(\omega Q P^{-1}\right)\right) .
$$

See e.g. Ara08-b, AM08-b, Gal02, Gal04, LLH96, Dor84, Ros69].

The concept of time operators was derived in the framework for the energy-time uncertainty relation in KKA4]. See also e.g. Fuj80, FWY80, GYS81-1, GYS81-2]. A strong connection with the decay of survival probability was pointed out by [Miy01, where the weak Weyl relation was introduced and then strong time operators were discussed. Moreover it was drastically generalized in Ara05 and some uniqueness theorems are established in Ara08.

This paper is inspired by [Miy01, Section VII] and [AM08-a]. In particular Arai and Matsuzawa AM08-a] developed machinery for reconstructing a pair of operators obeying the weak Weyl relation from a given pair $\left(H, T_{H}\right)$; in particular, they constructed a strong time operator associated with $\log |H|$. The main result of the paper is an extension of this work and we derive a strong time operator associated with general Hamiltonian $g(H)$ with a real-valued function $g$.

\subsection{Description of the main results}

By (1.1) a strong time operator $T_{P}$ associated with $P$ is given by

$$
T_{P}=Q .
$$

For the self-adjoint operator $(1 / 2) P^{2}$ in $L^{2}(\mathbb{R})$, it is established that

$$
T_{(1 / 2) P^{2}}=\frac{1}{2}\left(P^{-1} Q+Q P^{-1}\right)
$$

is an associated strong time operator referred to as the Aharonov-Bohm operator. Comparing (1.3) with (1.4) we arrive at

$$
T_{(1 / 2) P^{2}}=\frac{1}{2}\left(f^{\prime}(P)^{-1} T_{P}+T_{P} f^{\prime}(P)^{-1}\right),
$$

where $f(\lambda)=(1 / 2) \lambda^{2}$. We wish to extend formula (1.5) for more general $f$ 's and for any $\left(H, T_{H}\right)$. 
More precisely let $g$ be some Borel measurable function from $\mathbb{R}$ to $\mathbb{R}$. We want to construct a map $\mathscr{T}(g)$ such that $\mathscr{T}(g) T_{H}=T_{g(H)}$ and to show that

$$
T_{g(H)}=\frac{1}{2}\left(g^{\prime}(H)^{-1} T_{H}+T_{H} g^{\prime}(H)^{-1}\right) .
$$

We denote the set of $n$ times continuously differentiable functions on $\Omega \subset \mathbb{R}$ with compact support by $C_{0}^{n}(\Omega)$.

Proposition 1.2 Assume that $(H, T)$ satisfies the weak Weyl relation. Then

(1) $H$ has purely absolutely continuous spectrum. In particular $H$ has no point spectrum;

(2) $(H, \bar{T})$ also satisfies the weak Weyl relation.

Proof: (1) Refer to see Ara05. (2) It can be proven by a simple limiting argument. qed

Throughout, we suppose that the following assumptions hold.

Assumption $1.3(H, T)$ obeys the weak Weyl relation and $T$ is a closed symmetric operator.

Assumption 1.4 Let $g: \mathbb{R} \rightarrow \mathbb{R}$ be a Lebesgue measurable function such that

(1) $g \in C^{2}(\mathbb{R} \backslash K)$ for some closed subset $K \subset \mathbb{R}$ with Lebesgue measure zero;

(2) the Lebesgue measure of $\left\{\lambda \in \mathbb{R} \backslash K \mid g^{\prime}(\lambda)=0\right\}$ is zero.

We fix $(H, T), K \subset \mathbb{R}$ and $g \in C^{2}(\mathbb{R} \backslash K)$ satisfying Assumptions 1.3 and 1.4 in what follows. For a Lebesgue measurable function $f, f(H)$ is defined by

$$
f(H)=\int_{\operatorname{Spec}(H)} f(\lambda) d E_{\lambda}^{H}
$$

for the spectral resolution $E_{\lambda}^{H}$ of $H$. Let $Z$ be the set of singular points of $g^{-1}$ :

$$
Z=\left\{\lambda \in \mathbb{R} \backslash K \mid g^{\prime}(\lambda)=0\right\} \cup K,
$$

which is closed and has Lebesgue measure zero.

Now we will define a useful subspace $X_{n}^{\mathscr{D}}$. 
Definition 1.5 Let $\mathscr{D} \subset \mathscr{H}$ be a dense subspace. The subspace $X_{n}^{\mathscr{D}}, 0 \leq n \leq \infty$, in $\mathscr{H}$ is defined by

$$
X_{n}^{\mathscr{D}}=\text { linear hull of }\left\{\rho(H) \phi \mid \rho \in C_{0}^{n}(\mathbb{R} \backslash Z), \phi \in \mathscr{D}\right\},
$$

where $C_{0}^{0}=C_{0}$.

Lemma 1.6 $X_{n}^{\mathscr{D}}$ is dense in $\mathscr{H}$.

Proof: Let $(f, \Phi)=0$ for all $\Phi \in X_{n}^{\mathscr{D}}$. Then $\left(\rho(H)^{*} f, \phi\right)=0$ for all $\phi \in \mathscr{D}$ and $\rho \in C_{0}^{n}(\mathbb{R} \backslash Z)$, which implies that $f \in E_{Z}^{H} \mathscr{H}$, where $E^{H}$ denotes the spectral resolution of $H$. Since $H$ has purely absolutely continuous spectrum and the Lebesgue measure of $Z$ is zero, $f=0$ is concluded. Hence $X_{n}^{\mathscr{D}}$ is dense.

qed

The next proposition is fundamental.

Proposition 1.7 Ara05] Let $f \in C^{1}(\mathbb{R})$ and let both $f$ and $f^{\prime}$ be bounded. Then $f(H) \mathrm{D}(T) \subset \mathrm{D}(T)$ and

$$
T f(H) \phi=f(H) T \phi+i f^{\prime}(H) \phi, \quad \phi \in \mathrm{D}(T) .
$$

Proof: First suppose that $f \in C_{0}^{\infty}(\mathbb{R})$. Let $\check{f}$ denote the inverse Fourier transform of $f$. Then for $\psi \in \mathrm{D}(T)$,

$$
\begin{aligned}
(T \psi, f(H) \phi) & =(2 \pi)^{-1 / 2} \int_{\mathbb{R}}\left(T \psi, e^{-i \lambda H} \phi\right) \check{f}(\lambda) d \lambda \\
& =(2 \pi)^{-1 / 2} \int_{\mathbb{R}} \check{f}(\lambda)\left(\psi, e^{-i \lambda H}(T+\lambda) \phi\right) d \lambda=\left(\psi,\left(f(H) T+i f^{\prime}(H)\right) \phi\right) .
\end{aligned}
$$

So (1.7) follows for $f \in C_{0}^{\infty}(\mathbb{R})$. By a limiting argument on $f$ and the fact that $T$ is closed, (1.7) follows for $f \in C^{1}(\mathbb{R})$ such that $f$ and $f^{\prime}$ are bounded.

qed

This proposition suggests that informally

$$
T e^{-i t g(H)} \phi=e^{-i t g(H)} T \phi+t g^{\prime}(H) e^{-i t g(H)} \phi
$$

and then $T g^{\prime}(H)^{-1} e^{-i t g(H)} \phi=e^{-i t g(H)}\left(T g^{\prime}(H)^{-1}+t\right) \phi$. Symmetrizing $T g^{\prime}(H)^{-1}$, we expect that a strong time operator associated with $g(H)$ will be given by

$$
T_{g(H)}=\frac{1}{2}\left(g^{\prime}(H)^{-1} T+T g^{\prime}(H)^{-1}\right) .
$$


In order to establish (1.8), the remaining problem is to check the domain argument and to extend Proposition 1.7 for unbounded $f$ and $f^{\prime}$.

By the definition of $g$, for $\lambda \in \mathbb{R} \backslash Z$, there exists the derivative $d g(\lambda) / d \lambda=g^{\prime}(\lambda)$ and $g^{\prime}(\lambda)^{-1}<\infty$. Let

$$
\tilde{g}^{\prime}(\lambda)= \begin{cases}g^{\prime}(\lambda), & \lambda \notin Z \\ 0, & \lambda \in Z\end{cases}
$$

and define

$$
g^{\prime}(H)=\tilde{g}^{\prime}(H)
$$

Equivalently

$$
g^{\prime}(H)=\int_{\operatorname{spec}(\mathrm{H}) \backslash \mathrm{Z}} g^{\prime}(\lambda) d E_{\lambda}^{H} .
$$

In what follows we denote $g^{\prime}(\lambda)$ for $\tilde{g}^{\prime}(\lambda)$ without confusion may arise. Since the Lebesgue measure of $Z$ is zero and $H$ has purely absolutely continuous spectrum, we see that

$$
\operatorname{dim} \operatorname{ker} g^{\prime}(H)=0 \text {. }
$$

Thus $g^{\prime}(H)^{-1}$ is well defined.

Lemma 1.8 It follows that

(1) $T: X_{n}^{\mathrm{D}(T)} \rightarrow X_{n-1}^{\mathscr{H}}$ for $1 \leq n \leq \infty$.

(2) $g^{\prime}(H)^{-1}:\left\{\begin{array}{ll}X_{n}^{\mathscr{D}} \rightarrow X_{1}^{\mathscr{D}}, & 1 \leq n \leq \infty, \\ X_{0}^{\mathscr{D}} \rightarrow X_{0}^{\mathscr{D}}, & n=0,\end{array}\right.$ for any $\mathscr{D} \subset \mathscr{H}$.

Proof: Let $\Phi=\rho(H) \phi \in X_{n}^{\mathrm{D}(T)}$. By Proposition 1.7, $\Phi \in \mathrm{D}(T)$ and we have $T \Phi=i \rho^{\prime}(H) \phi+\rho(H) T \phi$. Then (1) follows. It is clear that $D\left(g^{\prime}(H)^{-1}\right) \ni \Phi=\rho(H) \phi$ and $g^{\prime}(H)^{-1} \Phi=\left(g^{\prime}(H)^{-1} \rho(H)\right) \phi$. Note that $\rho / g^{\prime} \in C_{0}^{1}(\mathbb{R} \backslash Z)$ for $\rho \in C_{0}^{n}(\mathbb{R} \backslash Z)$ with $n \geq 1$, and $\rho / g^{\prime} \in C_{0}(\mathbb{R} \backslash Z)$ for $\rho \in C_{0}(\mathbb{R} \backslash Z)$. Then (2) follows.

qed

Define the symmetric operator $\widetilde{D}$ by

$$
\widetilde{D}=\frac{1}{2}\left(g^{\prime}(H)^{-1} T+T g^{\prime}(H)^{-1}\right) \Gamma_{X_{1}^{\mathrm{D}(T)}} .
$$

$\widetilde{D}$ is well defined by Lemma 1.8. Actually $\widetilde{D}: X_{1}^{D(T)} \rightarrow X_{0}^{\mathscr{H}}$. Since the domain of the adjoint of $\widetilde{D}$ includes the dense subspace $X_{1}^{\mathrm{D}(T)}, \widetilde{D}$ is closable. We define

$$
D=\frac{1}{2} \overline{\left(g^{\prime}(H)^{-1} T+T g^{\prime}(H)^{-1}\right) \Gamma_{X_{1}^{\mathrm{D}(T)}}}
$$

The main theorem is as follows. 
Theorem 1.9 Suppose Assumptions 1.3 and 1.4. Then $(g(H), D)$ obeys the weak Weyl relation.

Example 1.10 Examples of strong time operators are as follows:

(1) Let $g$ be a polynomial. Then $Z=\left\{\lambda \in \mathbb{R} \mid g^{\prime}(\lambda)=0\right\}$ and a strong time operator associated with $g(H)$ is

$$
\frac{1}{2} \overline{\left(g^{\prime}(H)^{-1} T+T g^{\prime}(H)^{-1}\right) \Gamma_{X_{1}^{D(T)}}}
$$

(2) Let $g(\lambda)=\log |\lambda|$. Then $Z=\{0\}$ and a strong time operator associated with $\log |H|$ is

$$
\frac{1}{2} \overline{(H T+T H) \Gamma_{X_{1}^{\mathrm{D}(T)}}}
$$

This strong time operator is derived in [AM08-a.

(3) Let $(H, T)=(P, Q)$ and $g(\lambda)=\sqrt{\lambda^{2}+m^{2}}, m \geq 0$. Then $Z=\left\{\begin{array}{ll}\emptyset, & m>0 \\ \{0\}, & m=0\end{array}\right.$. A strong time operator associated with $H(P)=\sqrt{P^{2}+m^{2}}$ is

$$
\frac{1}{2} \overline{\left(H(P) P^{-1} Q+Q P^{-1} H(P)\right) \Gamma_{\mathrm{D}\left(X_{1}^{\mathrm{D}(Q)}\right)}} .
$$

$H(P)$ is a semi-relativistic Schrödinger operator.

(4) (3) can be generalized to fractional Schrödinger operators. Let $\alpha \in \mathbb{R} \backslash\{0\}$. Define $H_{\alpha}(P)$ by $H_{\alpha}(P)=\left(P^{2}+m^{2}\right)^{\alpha / 2}$. A strong time operator associated with $H_{\alpha}(P)$ is given by

$$
\frac{1}{2 \alpha} \overline{\left(\left(P^{2}+m^{2}\right) P^{-1} H_{\alpha}(P)^{-1} Q+Q H_{\alpha}(P)^{-1} P^{-1}\left(P^{2}+m^{2}\right)\right)\left\lceil_{\mathrm{D}\left(X_{1}^{\mathrm{D}(Q)}\right)}\right.} .
$$

\section{Proof of Theorem 1.9}

In order to prove Theorem 1.9 we prepare two lemmas, where it is proven that the weak Weyl relation holds for the pair $(g(H), \tilde{D})$ but on $X_{1}^{D(T)}$.

Lemma 2.1 Let $\Phi \in X_{1}^{\mathrm{D}(T)}$. Then

(1) $\Phi \in \mathrm{D}\left(g^{\prime}(H)^{-1}\right)$ and $g^{\prime}(H)^{-1} \Phi \in \mathrm{D}(T)$;

(2) $g^{\prime}(H)^{-1} e^{-i t g(H)} \Phi \in \mathrm{D}(T)$; 
(3) $e^{-i t g(H)} \Phi \in \mathrm{D}(T)$ and $T e^{-i t g(H)} \Phi \in \mathrm{D}\left(g^{\prime}(H)^{-1}\right)$.

(4) $e^{-i t g(H)} T \Phi \in \mathrm{D}\left(g^{\prime}(H)^{-1}\right)$;

Proof: Throughout the proof we set $\Phi=\rho(H) \phi \in X_{1}^{D(T)}$ with some $\rho \in C_{0}^{1}(\mathbb{R} \backslash Z)$ and $\phi \in D(T)$. Note that $g \in C^{2}(\mathbb{R} \backslash K)$.

(1) Since $\rho / g^{\prime} \in C_{0}^{1}(\mathbb{R} \backslash Z), g^{\prime}(H)^{-1} \Phi=\left(g^{\prime}(H)^{-1} \rho(H)\right) \phi \in \mathrm{D}(T)$ follows from Proposition 1.7.

(2) Since $e^{-i t g} \rho / g^{\prime} \in C_{0}^{1}(\mathbb{R} \backslash Z), e^{-i t g(H)} g^{\prime}(H)^{-1} \Phi \in \mathrm{D}(T)$ also follows from Proposition 1.7 .

(3) Since $\xi=e^{-i t g} \rho \in C_{0}^{1}(\mathbb{R} \backslash Z)$ and its derivative is bounded, $e^{-i t g(H)} \Phi \in \mathrm{D}(T)$ and

$$
T e^{-i t g(H)} \Phi=T \xi(H) \phi=\xi(H) T \phi+i \xi^{\prime}(H) \phi
$$

follows from Proposition [1.7. Here $\xi^{\prime}=-i t g^{\prime} e^{-i t g} \rho+e^{-i t g} \rho^{\prime} \in C_{0}(\mathbb{R} \backslash Z)$. From this we have $T e^{-i t g(H)} \Phi \in \mathrm{D}\left(g^{\prime}(H)^{-1}\right)$.

(4) Since $T \Phi=T \rho(H) \phi=i \rho^{\prime}(H) \phi+\rho(H) T \phi$ and then

$$
e^{-i t g(H)} T \Phi=i e^{-i t g(H)} \rho^{\prime}(H) \phi+e^{-i t g(H)} \rho(H) T \phi,
$$

we have $e^{-i t g(H)} T \Phi \in \mathrm{D}\left(g^{\prime}(H)^{-1}\right)$.

qed

Lemma 2.2 Let $\Phi \in X_{1}^{\mathrm{D}(T)}$. Then

$$
\tilde{D} e^{-i t g(H)} \Phi=e^{-i t g(H)}(\tilde{D}+t) \Phi .
$$

Proof: Let $\Phi=\rho(H) \phi \in X_{1}^{D(T)}$ with some $\rho \in C_{0}^{1}(\mathbb{R} \backslash Z)$ and $\phi \in D(T)$. We divide the proof into three steps.

(Step 1) It holds that

$$
T g^{\prime}(H)^{-1} e^{-i t g(H)} \Phi=e^{-i t g(H)}\left(T g^{\prime}(H)^{-1}+t\right) \Phi
$$

Proof: Let $\xi=e^{-i t g} \rho \in C_{0}^{1}(\mathbb{R} \backslash Z)$. As was seen in the proof of (3) of Lemma 2.1, both $\xi$ and $\xi^{\prime}$ are bounded and

$$
T e^{-i t g(H)} \Phi=T \xi(H) \phi=\xi(H) T \phi+i \xi^{\prime}(H) \phi
$$

Here

$$
\xi^{\prime}(H) \phi=-i t g^{\prime}(H) e^{-i t g(H)} \rho(H) \phi+e^{-i t g(H)} \rho^{\prime}(H) \phi .
$$


Then (2.3) and (2.4) yield that

$$
T e^{-i t g(H)} \Phi=t g^{\prime}(H) e^{-i t g(H)} \rho(H) \phi+e^{-i t g(H)}\left(\rho(H) T \phi+i \rho^{\prime}(H) \phi\right) .
$$

Note that $T \Phi=T \rho(H) \phi=\rho(H) T \phi+i \rho^{\prime}(H) \phi$. Then we have

$$
T e^{-i t g(H)} \Phi=e^{-i t g(H)}\left(T+t g^{\prime}(H)\right) \Phi
$$

Since we have already shown in (1) and (2) of Lemma 2.1 that $\Phi \in \mathrm{D}\left(g^{\prime}(H)^{-1}\right)$ and $g^{\prime}(H)^{-1} \Phi \in \mathrm{D}\left(e^{-i t g(H)} T\right) \cap \mathrm{D}\left(T e^{-i t g(H)}\right)$, we can substitute $g^{\prime}(H)^{-1} \Phi$ for $\Phi$ in (2.6) . Then (2.2) follows.

(Step2) It holds that

$$
g^{\prime}(H)^{-1} T e^{-i t g(H)} \Phi=e^{-i t g(H)}\left(g^{\prime}(H)^{-1} T+t\right) \Phi .
$$

Proof: Let $\Psi \in X_{1}^{\mathrm{D}(T)}$. (2.2) implies that

$$
\left(\Phi, T g^{\prime}(H)^{-1} e^{-i t g(H)} \Psi-e^{-i t g(H)} T g^{\prime}(H)^{-1} \Psi\right)=t\left(\Phi, e^{-i t g(H)} \Psi\right) .
$$

By (3) and (4) of Lemma 2.1, we can take the adjoint of both sides of (2.8). Then (2.7) follows if we transform $t$ to $-t$.

(Step3) Combining (2.2) and (2.7), we have (2.1).

qed

\section{Proof of Theorem 1.9:}

Let $\Phi \in \mathrm{D}(D)$. There exists $\Phi_{n} \in X_{1}^{\mathrm{D}(T)}$ such that $\Phi_{n} \rightarrow \Phi$ and $D \Phi_{n} \rightarrow D \Phi$ as $n \rightarrow \infty$ strongly. By Lemma 2.2. for each $\Phi_{n}, D e^{-i t g(H)} \Phi_{n}=e^{-i t g(H)}(D+t) \Phi_{n}$ holds. Since $D$ is closed, the theorem follows by a limiting argument.

qed

Acknowledgments: We thank A. Arai for helpful comments and careful reading of the first manuscript. We also thank unknown referee for useful comments.

\section{References}

[Ara05] A. Arai, Generalized weak Weyl relation and decay of quantum dynamics, Rev. Math. Phys. 17 (2005), 1071-1109.

[Ara08] A. Arai, On the uniqueness of weak Weyl representations of the canonical commutation relation, to be published in Lett. Math. Phys.

[Ara08-b] A. Arai, Necessary and sufficient conditions for a Hamiltonian with discrete eigenvalues to have time operators, mp-arc 08-154, preprint 2008.

[AM08-a] A. Arai and Y. Matsuzawa, Construction of a Weyl representation from a weak Weyl representation of the canonical commutation relation, Lett. Math. Phys. 83 (2008), 201-211. 
[AM08-b] A. Arai and Y. Matsuzawa, Time operators of a Hamiltonian with purely discrete spectrum, to be published in Rev. Math. Phys.

[Gal02] E. A. Galapon, Self-adjoint time operator is the rule for discrete semi-bounded Hamiltonians, Proc. R. Soc. Lond. A 458 (2002), 2671-2689.

[Gal04] E. A. Galapon, R. F. Caballar and R. T. Bahague Jr, Confined quantum time of arrivals, Phys. Rev. Lett.93 (2004), 180406.

[Dor84] G. Dorfmeister and J. Dorfmeister, Classification of certain pairs of operators $(P, Q)$ satisfying $[P, Q]=-i \mathrm{Id}$, J. Funct. Anal. 57 (1984), 301-328.

[Fuj80] I. Fujiwara, Rational construction and physical signification of the quantum time operator, Prog. Theor. Phys. 64 (1980), 18-27.

[FWY80] I. Fujiwara, K. Wakita and H. Yoro, Explicit construction of time-energy uncertainty relationship in quantum mechanics, Prog. Theor. Phys. 64 (1980), 363-379.

[GYS81-1] T. Goto, K. Yamaguchi and N. Sudo, On the time opertor in quantum mechanics, Prog. Theor. Phys. 66 (1981), 1525-1538.

[GYS81-2] T. Goto, K. Yamaguchi and N. Sudo, On the time opertor in quantum mechanics. II, Prog. Theor. Phys. 66 (1981), 1915-1925.

[KA94] D. H. Kobe and V. C. Aguilera-Navarro, Derivation of the energy-time uncertainty relation. Phys. Rev. A 50 (1994), 933 - 938.

[LLH96] H. R. Lewis, W. E. Laurence and J. D. Harris, Quantum action-angle variables for the harmonic oscillator, Phys. Rev. Lett. 26 (1996), 5157-5159.

[Miy01] M. Miyamoto, A generalised Weyl relation approach to the time operator and its connection to the survival probability, J. Math. Phys. 42 (2001), 1038-1052.

[Ros69] D. M. Rosenbaum, Super Hilbert space and the quamntum-mechanical time operators, J. Math. Phys.19 (1969), 1127-1144. 